\title{
Real-time Detection of False Data Injection in Smart Grid Networks: An Adaptive CUSUM Method and Analysis
}

\author{
Yi Huang ${ }^{\dagger}$, Jin Tang*, Yu Cheng*, Husheng Li ${ }^{\ddagger}$, Kristy A. Campbell ${ }^{\S}$, and Zhu Han ${ }^{\dagger}$ \\ ${ }^{\dagger}$ Department of Electrical and Computer Engineering, University of Houston, Houston, TX, USA \\ *Department of Electronics and Computer Engineering, Illinois Institute of Technology, Chicago, IL, USA \\ ${ }^{\ddagger}$ Electrical Engineering and Computer Science Department, University of Tennessee, Knoxville, TN, USA

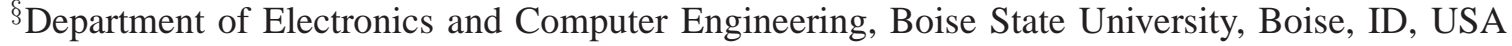

\begin{abstract}
Smart grid is delay-sensitive and requires the techniques that can identify and react on the abnormal changes (i.e. system fault, attacker, shortcut, etc.) in a timely manner. In this paper, we propose a real-time detection scheme against false data injection attack in smart grid networks. Unlike the classical detection test, the proposed algorithm is able to tackle the unknown parameters with low complexity and process multiple measurements at once, leading to a shorter decision time and better detection accuracy. The objective is to detect of adversary as quickly as possible while satisfy certain detection error constraints. A Markov chain based analytical model is constructed to systematically analyze the proposed scheme. With the analytical model, we are able to configure the system parameters for guaranteed performance in terms of false alarm rate, average detection delay, and missed detection ratio under a detection delay constraint. The Simulations are conducted with MATPOWER 4.0 package for different IEEE test systems.
\end{abstract}

\section{INTRODUCTION}

The smart grid has improved the robustness and efficiency of traditional power grid networks by exploiting the modern technologies. In particular, information exchange among users, operators, and control devices significantly improves the efficiency in production, transmission, and distribution. However, integration of intelligence into the power grid needs to act punctually on abnormal situations (i.e. system fault, attacks, shortcut, etc.) [1].

Indeed, smart grid is delay-sensitive and requires the techniques that can identify and react on the abnormal changes in a timely manner. If the detection and responses are not made promptly, the grid may become unstable and further cause the catastrophic failures over the entire network. For example, in the control center of smart grid, an essential task of the energy management system (EMS) is to estimate the system states by collecting data from remote meters periodically. If the adversaries are able to inject malicious data, EMS may produce the false state estimation, which potentially results in wrong decisions on billing, power dispatch, erroneous analysis and even blackout [2]. Thus, the smart grid network must incorporate the protection mechanism, which has the capability of detecting the abnormal change and then making the decision as quickly as possible. Such an issue strongly motivates us to propose the quick detection based detection scheme.

This work is partially supported by US National Science Foundation CNS0953377, CNS-0905556, CNS-0910461, ECCS-1028782, and CNS 1117560.
There are many studies on smart grid security in the literature. A framework for analyzing the impact of cyberattacks in a smart grid was presented in [3], [4]. The work in [5]-[7] formulated the attack that are able to evade from the conventional detection in smart grid. The false data injections is studied in [8]-[10] as one type of the cyber-attacks in the power system. Authors in [11] discovered the microgrid vulnerability in the smarter power system under the false data injection attack. In [12], the false data injection attacks is shown to interrupt the energy routing process. In this paper, we like to focus on studying in the observable context with the proposed detection scheme that can be an interesting practical contribution for smart grid networks.

To address the false data injection attacks in the smart grid, EMS in the control center needs to be equipped with the capability of real-time detection of malicious attacks by analyzing the statistical behavior of the state estimation process. According to the quickest detection (QD) framework [13], the cumulative sum (CUSUM) based approach fits well to this type of detection problems because of its non-Bayesian properties. Such a framework aims to determine a change of the observed statistics as quickly as possible based on online observations, the user-defined decision rules, and the requirement of detection accuracy. The decision rules should be properly designed to optimize the tradeoff between the stopping time and decision accuracy.

The QD technique is normally combined with the statistical hypotheses test (SHT) [14], [15]. The mechanism of SHT is that the receiver classifies a sequence of observations into one of the candidate hypotheses; a hypothesis normally represents a type of distributions. The QD and SHT have been applied to a variety of networks. The authors in [16] used the CUSUM tests as a collaborative QD for detecting a distribution change in ad hoc networks. The authors in [17] utilized the CUSUM test to address the real-time backoff misbehavior problem in IEEE 802.11 based wireless networks. However, no much existing work has considered the unique environment of smart grid networks.

In this paper, a countermeasure strategy of the false data injection attack is considered in the form of adversary detection. The problem formulation of detecting the false data injection is based on the bad data detection (BDD) for the smart grid state estimation. The proposed scheme is able to determine the existence of adversary as quickly as possible without violating the given constraints such as a certain level of detection 
accuracy in terms of the false alarm rate and missed detection rate. In [18], we studied some preliminary works that includes the basic mathematic derivation and numerical simulations; without loss of generality, one in conference version is motivated on the straightforward approach via directly evaluating the likelihood of load for detection decision, instead of formulating the algorithm based on the likelihood of residual in this paper, i.e., state estimation in power systems is based on measurement of residual, and therefore, the derivation and result from this journal can be more accurate and practical for real world applications. In additions, the conference one measured a limited range of the unknown via utilizing oneside Rao test for simplicity, while this paper considers the quadratic equivalence of Rao test for solving the unknown. Essentially, this journal focuses on the thorough examination for the proposed algorithm in terms of analytical model and performance simulations. The development of an analytical model in this paper for the proposed algorithm provides theoretical guidance for quantitative performance analysis, and it further makes available the precious insight on system parameters configuration for guaranteed performance in terms of fundamental performance metrics. The main contributions are as follows:

- We develop a framework for real-time detection of false data injection attacks in the smart grid network, under certain detection quality constraints. While the conventional state estimation [19], [20] for bad data detection focuses on balancing between the false alarm rate and missing detection ratio, our approach aims to minimize the detection delay under the error probability constraint. In addition, the conventional approach makes decisions based on snapshot measurements only, but the proposed framework analyzes a sequence of samples for more reliable decisions over time.

- The proposed algorithm is able to detect the presence of false data attacks in that the probability density function of the post-change is unknown due to the unknown parameters. However, the classical CUSUM test assumes the perfect knowledge of the likelihood functions. While the existing generalized likelihood ratio test (GLRT) approach can resolve the unknown parameters, it has high complexity. This paper proposes a new low complexity approach with shorter decision delay and more accurate decision, which is asymptotically equivalent to the GLRT test.

- An analytical model for the proposed algorithm is developed, which provides the theoretical guidance for quantitative performance analysis. With the analytical model, it gives the insight on system parameters configuration for the on-line detection of false data injection attack. System parameters can also be computed for guaranteed performance in terms of three fundamental performance metrics: the false alarm rate, average detection delay, and missed detection ratio under a detection delay constraint. In other words, our analytical model can guide us to configure a detection system based on some detection performance requirement.
- The performance of the proposed algorithm is evaluated by both mathematic analysis and simulations. Notes that simulations are conducted under MATPOWER 4.0 package [21] for different IEEE test systems to ensure the experiment accuracy and proficiency.

\begin{tabular}{ll}
\hline Notation & \multicolumn{1}{c}{ Description } \\
\hline EMS & energy management system \\
QD & quickest detection \\
CUSUMcumulative sum \\
SHT & statistical hypothesis test \\
BDD & bad data detection \\
AGC & automatic generation control \\
OPF & optimal power flow \\
ARL & average run length \\
GLRT & generalized likelihood ratio test \\
TPM & transition probabilities matrix \\
FAR & false alarm rate \\
MDR & misssed detection ratio \\
$B$ & number of buses in power system \\
$C$ & detection delay constraint \\
$\mathcal{H}_{e}$ & hypothesis $e$ in SHT \\
$V_{q}$ & voltage measurement at the bus $q$ \\
$\theta_{q}$ & phase measurement at the bus $q$ \\
$X_{q r}$ & reactance between bus $q$ and $r$ \\
$M_{q r}$ & power flow measurement from bus $q$ to $r$ \\
$M_{q}$ & power injection measurement at bus $q$ \\
$h$ & detection threshold, a function of error probability \\
$n$ & observation index \\
$m$ & total number of active power measurement \\
$\mathbf{Z}$ & a vector of power measurement $\left(M_{q} r, M_{q}\right.$, or both) \\
$\mathbf{x}$ & the unknown state vector for state estimation \\
$\mathbf{e}$ & a vector of measurement noise \\
$\mathbf{H}$ & Jacobian matrix \\
$T_{D}$ & detection delay for the proposed algorithm \\
$T_{h}$ & the moment when detector raises the alarm \\
$\tau$ & the moment when adversary initializes the attack \\
$S_{n}$ & CUSUM statistic at observation index $n$ \\
$\mathbf{P}$ & the transition probability matrix for Markov chain \\
$\pi_{i}^{0}$ & the steady state probability that a detector starts \\
$\pi_{i}$ & the steady state probability that a detector is \\
\hline & at state $i$ \\
for
\end{tabular}

THE DESCRIPTION OF SOME IMPORTANT SYMBOLS AND ABBREVIATIONS.

The remainder of this paper is organized as follows. Section II describes the system model. Section III resents and analyzes the newly proposed scheme, the adaptive CUSUM algorithm. Section IV develops the Markov chain based the analytical model. Section V presents extensive numerical and simulation results for performance evaluation. Section VI gives the concluding remarks. Table I includes some important notations used in this paper.

\section{Problem Formulation}

Figure 1 illustrates the IEEE 4-buses test system with 2 generators; each bus has its corresponding voltage $\left(V_{q}\right)$ and phase angle $\left(\theta_{q}\right)$; the control center sends the power measurement data $\left(z_{q r}\right)$ to the state estimator which generate an estimate of system state to be used in different functions such as the automatic generation control (AGC), optimal power flow (OPF), or EMS. The operator makes the final decision on generator control and load management.

As an essential role in the power system, the state estimator uses the steady-state system model to estimate the system 
status (i.e. the voltages at all buses over the time) [22]. Speaking in general, state estimation with a total of $B$ active buses in a practical power system can be described as

$$
\mathbf{Z}=\mathbf{h}(\mathbf{x})+\mathbf{e},
$$

where $\mathbf{Z}$ denotes the measurement data, $\mathbf{x}$ represents the unknown state including the voltage level $V_{q}$ and the phase angel $\theta_{q}$ of each bus $q \in B$, and $\mathbf{e}$ is the Gaussian measurement noise with a zero mean and a covariance matrix $\boldsymbol{\Sigma}_{e}$. Noticing that a nonlinear $\mathbf{h}(\mathbf{x})$ is determined by the network topology, the real power flow from bus $q$ to bus $r$ can be expressed as

$M_{q r}=V_{q}^{2}\left(g_{s q}+g_{q r}\right)-V_{q} V_{r}\left(g_{q r} \cos \theta_{q r}+b_{q r} \sin \theta_{q r}\right)$,

$\tilde{M}_{q r}=-V_{q}^{2}\left(b_{s q}+b_{q r}\right)-V_{q} V_{r}\left(g_{q r} \cos \theta_{q r}-b_{q r} \sin \theta_{q r}\right),(2)$

where the admittance of the series branch between buses $q$ and $r$ is $\left(g_{q r}+j b_{q r}\right)$, and the admittance of the shunt branch at bus $q$ is $\left(g_{s q}+j b_{s q}\right)$. The formulations of real and reactive power injection can be constructed in the similar way such as described in (2).

For simplicity, the linear state estimation model is applied in this paper. Notice that all shunt elements, bus, branch and reactive power flow are neglected and the bus voltage magnitude is known [19]. The power flow and power injection can be linearized and described as

$$
\begin{array}{rcc}
M_{q r} & =\quad \frac{\theta_{q r}}{X_{q r}}, \\
M_{q} & =\sum_{r \in B_{q}} M_{q r},
\end{array}
$$

where $M_{q}$ is denoted to the power injection, $B_{q}$ is the set of bus numbers that are directly connected to bus $q, X_{q r}$ is the reactance between bus $q$ and bus $r$. Further, we can simplify ${ }^{1}$ (1) to

$$
\mathbf{Z}_{n}=\mathbf{H x}+\mathbf{e}_{n},
$$

where $\mathbf{H}$ is the constant Jacobian matrix, $\mathbf{Z}_{n}=$ $\left[Z_{n, 1}, \cdots, Z_{n, m}\right]^{T}$ with $m$ measurements at the observation index $n \in 1,2,3, \cdots$, and $\mathbf{x}=\left[\theta_{2}, \ldots, \theta_{B}\right]^{T}$. Notice that phase angle $\theta_{0}$ for bus 0 is assumed known as a reference angle, and the size of $\mathbf{Z}_{n}$ is normally greater than that of $\mathbf{x}$. [19], [23] One objective of (4) is to determine the $\hat{x}$ which can minimize

$$
\left(\mathbf{Z}_{n}-\mathbf{H} \hat{\mathbf{x}}\right)^{T} \boldsymbol{\Sigma}_{e}^{-1}\left(\mathbf{Z}_{n}-\mathbf{H} \hat{\mathbf{x}}\right)
$$

By applying the weighted least square, the estimated system state $\hat{\mathbf{x}}$ is:

$$
\hat{\mathbf{x}}=\left(\mathbf{H}^{T} \boldsymbol{\Sigma}_{e}^{-1} \mathbf{H}\right)^{-1} \mathbf{H}^{T} \boldsymbol{\Sigma}_{e}^{-1} \mathbf{Z}_{n} .
$$

For BDD system, we compare the power-flow measurements $\mathbf{Z}_{n}$ with the estimated active power-flow $\hat{\mathbf{Z}}_{n}$ by the phase angle estimate $\hat{\mathbf{x}}$. $\hat{\mathbf{Z}}_{n}$ can be written as:

$$
\hat{\mathbf{Z}}_{n}=\mathbf{H} \hat{\mathbf{x}}=\mathbf{H}\left(\mathbf{H}^{T} \boldsymbol{\Sigma}_{e}^{-1} \mathbf{H}\right)^{-1} \mathbf{H}^{T} \boldsymbol{\Sigma}_{e}^{-1} \mathbf{Z}_{n}=\Im \mathbf{Z}_{n},
$$

\footnotetext{
${ }^{1}$ The DC model is adopted due to practical security constraint unit commitment (SCUC) and market operations Most of the control centers use linear power model for state estimation because of two reasons. First, the phase differences are relatively small so that linear model can be employed. Second, due to the complexity of computing AC model, the linear model is used for real-time analysis in the power system operation [24]
}

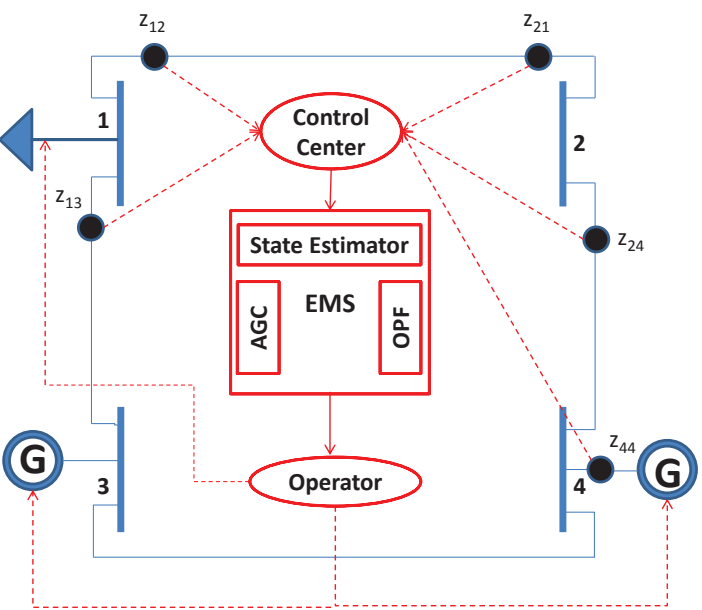

Fig. 1. An illustration of the 4 bus power network, control center, a few main functions (AGC, OPF, EMS), and the operator. Notes that "G" represents as the generators, the black dot represents available active power-flow measurements, and the triangular on the bus represents the load of the region or the city.

where $\Im$ is known as the hat matrix. Define the residue vector as

$$
\mathbf{R}_{n}=\mathbf{Z}_{n}-\hat{\mathbf{Z}}_{n}
$$

The expected value and the covariance of residual $\mathbf{R}_{n}$ are

$$
E\left(\mathbf{R}_{n}\right)=\mathbf{0},
$$

and

$$
\boldsymbol{\Sigma}_{\mathbf{R}}=\left[\mathbf{I}-\mathbf{H}\left(\mathbf{H}^{T} \boldsymbol{\Sigma}_{e}^{-1} \mathbf{H}\right)^{-1} \mathbf{H}^{T} \boldsymbol{\Sigma}_{e}^{-1}\right] \boldsymbol{\Sigma}_{e},
$$

respectively. The system can perform BDD by analyzing $\mathbf{R}_{n}$ [19].

In brief, the conventional state estimation for false data injection detection uses only snapshot measurements, and therefore, we like to apply the online quickest detection technique using a sequence of measurements for more reliable decisions.

\section{ADAPTIVE CUSUM ALGORITHM}

In this paper, we propose an adaptive CUSUM algorithm for real-time detection of false data attacks in smart grid state estimation. The proposed scheme evaluates the measurements before the potential bad data is removed by BDD. The detection system formulation as presented in [13] [25] is no longer useful in the scenario under our consideration, because unknown parameters exist in the post-change distribution and may dynamically change over the detection process. Our main motivation is to derive a detection model considering the existence of the unknown, and then develop an analytical model that can guide us configure the detection system for guaranteed performance. The proposed scheme does not require the Maximum Likelihood (ML) estimate of the unknown, thereby making the computation process much simpler.

Under a false data injection attack, the false data $\mathbf{b}_{n}$ is maliciously injected into the power flow measurement vector as

$$
\mathbf{Z}_{n}=\mathbf{H x}+\mathbf{b}_{n}+\mathbf{e}_{n} .
$$

Residual vector $\mathbf{R}_{n}$ can be well approximated by a Gaussian random variable because of Gaussian thermal measurement 
noise $\mathbf{e}_{n}$ [26]. When there is no attack, the residual vector $\mathbf{R}_{n}$ follows Gaussian distribution $\mathcal{N}\left(\mathbf{0}, \boldsymbol{\Sigma}_{\mathbf{R}}\right)$. Under attack, $\mathbf{R}_{n}$ follows $\mathcal{N}\left(\mathbf{a}_{n}, \boldsymbol{\Sigma}_{\mathbf{R}}\right)$, where

$$
\mathbf{a}_{n}=\mathbf{K} \mathbf{b}_{n},
$$

where $\mathbf{K}=(\mathbf{I}-\Im)$. Notice that $\mathbf{a}_{n}=$ $\left[a_{n, 1}, a_{n, 2}, \cdots, a_{n, m}\right]^{T}, \in \mathbb{R}^{m}$ is not known a priori(i.e., the adversary's statistical model, attack patterns, or mathematical distributions can not be known in advance. This issue will be addressed later in this section.) Then, we have the binary hypothesis as

$$
\begin{cases}\mathcal{H}_{0}: & \mathbf{R}_{n} \sim \mathcal{N}\left(\mathbf{0}, \boldsymbol{\Sigma}_{\mathbf{R}}\right), \\ \mathcal{H}_{1}: & \mathbf{R}_{n} \sim \mathcal{N}\left(\mathbf{a}_{n}, \boldsymbol{\Sigma}_{\mathbf{R}}\right),\end{cases}
$$

and assumes the false data injection becomes active at randomtime moment $\tau$. In other words, a change of the distribution from $\mathcal{N}\left(\mathbf{0}, \boldsymbol{\Sigma}_{\mathbf{R}}\right)$ to $\mathcal{N}\left(\mathbf{a}_{n}, \boldsymbol{\Sigma}_{\mathbf{R}}\right)$ at $\tau$. Notes that we process the measurement data before a BDD removes the potential residual.

We denote $T_{h}$ as the stopping time for declaring the best arm under current observation. $\tau$ is a change time. In other words, it is the switch point from one distribution belongs to the normal state to another distribution under the attack. Based on the Lorden's formulation [13], we minimize the worst case of detection delay, which can be described as:

$$
T_{D}=\inf _{T_{h} \in \mathcal{T}} \sup \operatorname{esssup} E_{\tau}\left[\left(T_{h}-\tau+1\right)^{+} \mid \mathcal{F}_{\tau-1}\right],
$$

which $\tau>1, \mathcal{F}_{\tau}$ denotes the smallest $\alpha$-field with respect to the observations, $\mathcal{T}$ is the set of all stopping time with respect to $\mathcal{F}_{\tau}$, and $E_{\tau}$ is the expectation that the change time is $\tau$. However, most CUSUM-based models assume the perfect knowledge of the likelihood functions [25]. In the scenario of intrusion detection in smart grid state estimation, the variable from the $\mathcal{H}_{1}$ distribution cannot be completely defined because of the unknown. The detection also needs to address the issue that multiple measurements are correlated each together in a single online observation. Thus, we need to employ the technique to solve the issues for real-time detection of false data injection in smart grid networks.

The proposed quickest detection algorithm is recursive in nature, and each recursion comprises two interleaved steps: i) unknown variable solver based on Rao test and ii) multi-thread CUSUM test. The proposed CUSUM algorithm updates a likelihood ratio term based on a series of power measurements with a stopping time $T_{h}$, describes as:

$$
T_{h}=\inf \left\{n \geq 1 \mid S_{n}>h\right\},
$$

where the detection threshold $h$ is a function of false alarm rate (FAR), and its value is determined numerically. We will discuss how to determine the value of $h$ in Section IV. At the $n^{t h}$, the cumulative statistic $S_{n}$ can be solved recursively and described as:

$$
S_{n}=\max \left[0, S_{n-1}+L_{n}\right],
$$

where the $S_{n}$ returns to zero for statistical accuracy if its value is negative, $S_{0}=0$ initially, and

$$
L_{n}=\log \frac{f_{1}\left(\mathbf{R}_{n}\right)}{f_{0}\left(\mathbf{R}_{n}\right)},
$$

being the likelihood ratio function based on the $n^{\text {th }}$ round of measurement denoted as the observation vector $\mathbf{R}_{n}\left(R_{n, l}, l \in\right.$ $1,2, \cdots, m)$. In (16), $f_{1}\left(\mathbf{R}_{n}\right)$ is the distribution associated with the hypothesis $\mathcal{H}_{1}$ with false data injection, and $f_{0}\left(\mathbf{R}_{n}\right)$ is the distribution associated with the hypothesis $\mathcal{H}_{0}$ in the normal state. Therefore, the control center is able to declare the alarm when the accumulation crosses a certain threshold $h$, the cumulative process is terminated, and average run length (ARL) is equivalent to $T_{h}$.

As the value of $\mathbf{a}_{n}$ in (11) is unknown, the author in [27] proposed to implement the generalized likelihood ratio test (GLRT) in the Page's CUSUM algorithm with the unknown. The idea is to apply likelihood ratio test (LRT) by replacing the unknown with the ML estimation. The GLRT approach is asymptotically minimax, and can be written as

$$
S_{n}=\min _{1 \leq n \leq T_{h}} \max _{a_{n}} \sum_{i=n}^{T_{h}} \log \frac{f_{1}\left(\mathbf{R}_{i} \mid \mathbf{a}_{i}\right)}{f_{0}\left(\mathbf{R}_{i}\right)} .
$$

In other words, we minimize the effect of the unknown while considering the worst case situation (i.e. the second maximization in (17)). Thus, by applying GLRT in the CUSUM algorithm, we can ensure a certain level of detection accuracy for QD, while minimizing the potential effect from the unknown in the system. However, the recursive expression of (17) for the CUSUM test is no longer available as shown in (15). It is because GLRT needs to compute every unknown element of $\mathbf{a}_{n}$ based on samples up to the current observation $n$. In other words, GLRT approach requires storing the estimated data and ML-estimating the unknown at every point. Thus, in practice, the GLRT is too difficult from the view points of hardware and software implementation. Moreover, the work in [28] states that Rao test might be more robust but less complex than the GLRT real operating situations. In [29], the performance of Rao test based detectors performs better than GLRT in parameter estimation and handling training-free scenario.

For the multi-thread CUSUM algorithm, the desired approach is to solve the unknown recursively, avoiding ML estimation. Thus, we consider the Rao test [30], which is asymptotically equivalent to the GLRT. The derivation of Rao test is similar to the locally most powerful (LMP) test but much simpler. The Rao test has the straight-forward calculation by taking derivative of $L_{n}$ with respect to the unknown evaluated around the region of interests. In our case, we analyze the case where the region is around zero due to the hypothesis $\mathcal{H}_{0}$ has zero mean. The statistic [30] of the Rao test for detection can be modified and rewritten as follows at observation $n$ :

$$
\mathcal{I}\left(\mathbf{R}_{n}\right)=\left.\left.\frac{\partial L_{n}}{\partial \mathbf{a}_{n}}\right|_{\mathbf{a}_{n}=\mathbf{0}} ^{T}\left[\left.\mathbf{J}^{-1}\left(\mathbf{a}_{n}\right)\right|_{\mathbf{a}_{n}=\mathbf{0}}\right] \frac{\partial L_{n}}{\partial \mathbf{a}_{n}}\right|_{\mathbf{a}_{n}=\mathbf{0}},
$$

where $\mathbf{J}$ is the Fisher information matrix [31]. By inspecting (18) and evaluating (11)-(12), we notice that the computation of the inverse Fisher information matrix can be simplified and equivalent to the covariance of residual.

Based on (12), we can write the binary hypothesis $\left\{\mathcal{H}_{0}, \mathcal{H}_{1}\right\}$ by expanding the multivariate normal distributions. Next, we 
apply (18) to (16) by taking its derivative with respect to $\mathbf{a}_{n}$ evaluated at $\mathbf{a}_{n}=\mathbf{0}$. Finally, by recursion, the multi-thread CUSUM-based statistic can be described as follows:

$$
S_{n}=\max \left\{0, S_{n-1}+\mathcal{I}\left(\mathbf{R}_{n}\right)\right\}
$$

where $\mathcal{I}\left(\mathbf{R}_{n}\right)=\left[\left(\mathbf{R}_{n}^{T} \boldsymbol{\Sigma}_{\mathbf{R}}^{-1}\right)^{T}+\boldsymbol{\Sigma}_{\mathbf{R}}^{-1} \mathbf{R}_{n}\right]^{T} \boldsymbol{\Sigma}_{\mathbf{R}}\left[\left(\mathbf{R}_{n}^{T} \boldsymbol{\Sigma}_{\mathbf{R}}^{-1}\right)^{T}+\right.$ $\left.\Sigma_{\mathbf{R}}^{-1} \mathbf{R}_{n}\right]$. Notice that the cumulative statistic is now independent from the unknown variable, and (19) becomes a scalar quantity once it is computed. In summary, the control center observes actual power-flow measurements and generates the vector of residual from $m$ measurement samples taken in the $n^{t h}$ round of observation. The proposed scheme composes with two interleaved steps: the unknown variable solver and multi-thread CUSUM test. The control center will monitor the CUSUM statistic in (19) against the threshold to detect the false data injection attacks. The alarm rises when the CUSUM statistic $S_{n}$ exceeds the threshold. The framework of the adaptive CUSUM algorithm of the proposed scheme is shown in Algorithm 1.

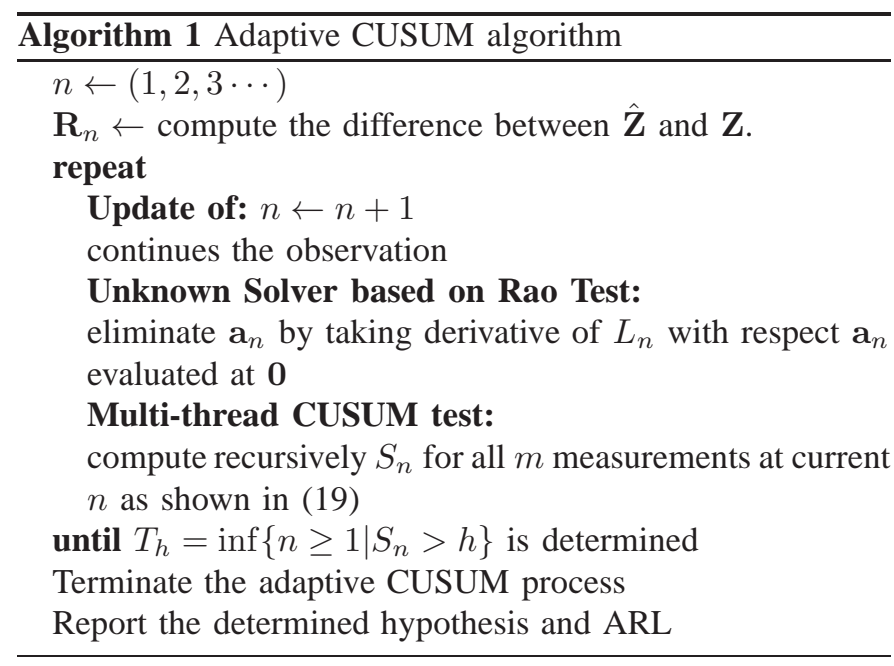

\section{Markov Chain Based Analytical Model}

In this section, we develop the Markov chain based analytical model to systematically examine the proposed scheme for the false data injection attack. The Markov chain based model produces quantitative performance analysis, and provides theoretical guidance on the system configuration for performance guarantee in terms of three fundamental performance metrics: the expectation of false-alarm rate, the expectation of missingdetection rate, and the expectation of detection delay.

\section{A. Analysis Model}

For analysis purpose, we discretize $\mathbb{R}^{+} \bigcup 0$ into the finite sets $\left\{U_{1}, \cdots, U_{F-1}, U_{F}\right\}$, where $U_{1}=0$, and $U_{F}$ is the set whose value is greater than or equal to $h$. In other words, $F$ is the total number of transition from 0 to the state that has the value greater than or equal to $h$. There are several approaches for discretization [32] [33]. In this paper, we employ uniform sampling without loss of generality. Alternative discretization methods can also be employed like the $\mu$-law or $A$-law in the pulse-code modulation. Moreover, from (19), we know that the sequence exhibits the Markov property, which the current state $j=S_{n}$ at observation $n$ only depends on the previous state $i=S_{n-1}$ at $n-1$, but not on the past history [34].

The transition probabilities of the Markov chain for the proposed scheme from state $i$ at $(n-1)$ to state $j$ at $n$ can be described as

$$
\begin{aligned}
& P_{i j}=P\left(S_{n}=j \mid S_{n-1}=i\right), \text { under } \mathcal{H}_{0} ; \\
& \hat{P}_{i j}=P\left(S_{n}=j \mid S_{n-1}=i\right), \text { under } \mathcal{H}_{1} .
\end{aligned}
$$

Note that The Markov chain based analytical model for the proposed scheme involves two different transition probabilities matrix (TPM): one is under the normal state environment; and the other one is under the false data attack. The normal TPM can help determining the initial state as well as false alarm rate. With the initial states, the average detection delay and detection delay can be analyzed by using the TPM under attack. We can calculate TPMs: $\mathbf{P}$ and $\hat{\mathbf{P}}$ with the size of $(F+1) \times(F+1)$, under the hypothesis $\mathcal{H}_{0}$ and $\mathcal{H}_{1}$ according to $f_{0}\left(\mathbf{R}_{n}\right)$ and $f_{1}\left(\mathbf{R}_{n}\right)$, respectively. Here, we assume that the attacker's strategy is stationary. If the attackers' attack has zero mean but nonzero variance, the hypothesis test problem becomes detecting the different variances with vs. without attack. If the attackers' attack has nonzero mean and nonzero variance, the hypothesis test has two dimensions (mean and variance). Both cases can be investigated by a similar way to our current analysis (attacker have nonzero mean and zero variance). Due to page limit, we leave this for the future study.

The initial steady state probability of the Markov chain, which the process starts from a normal state, can be determined as:

$$
\pi_{j}^{0}=\frac{\pi_{j}}{\sum_{i=0}^{F-1} \pi_{j}}, \quad \text { given } \quad j \in\left\{0, U_{1}, \cdots, U_{F-1}\right\},
$$

and the steady-state probability can be determined:

$$
\pi_{j}=\sum_{i=0}^{F} P_{i j} \pi_{i}
$$

where $j \in\left\{0, U_{1}, \cdots, U_{F}\right\}$ and $\sum_{j=0}^{F} \pi_{j}=1$.

Next, based on the Markov chain model, we study the theoretical performance analysis of detection delay, false alarm rate and missed detection ratio expectations, respectively, in the following subsections.

\section{B. The Expectation of Detection Delay}

To determine the expectation $\left(E_{\hat{\mathbf{P}}}\left[T_{D}\right]\right)$ of detection delay, we utilize the weighted average of the expected number of transitions from every initial state $\left(\pi_{0}^{0}, \pi_{1}^{0}, \cdots, \pi_{F-2}^{0}, \pi_{F-1}^{0}\right)$ to state $U_{F}$ based on $\hat{\mathbf{P}}$. We set $\Omega_{g F}, g \in\left\{0, U_{1}, \cdots, U_{F-1}\right\}$ as the expected number of transitions for state $g$ to state $U_{F}$. Following the derivation from [34], the numerical value of $\Omega_{i F}$ can be determined as follows:

$$
\Omega_{i F}=1+\sum_{g \neq F} \hat{P}_{i g} \Omega_{g F},
$$

where the transition probability $\hat{P}_{i g} \in \hat{\mathbf{P}}$ is from state $i$ to state $g$. The expectation of detection delay can be obtained 
from the results of (21) and (23):

$$
E_{\hat{\mathbf{P}}}\left[T_{D}\right]=\sum_{i=0}^{F-1} \pi_{i}^{0} \Omega_{i F} .
$$

\section{The Expectation of False Alarm Rate}

The expectation $\left(E_{\mathbf{P}}[\mathrm{FAR}]\right)$ of false alarm rate is the probability that the proposed CUSUM statistic $S_{n}$ reaches to the state $U_{F}$ when there is no attacker in the network. As described in [34], $E_{\mathbf{P}}[\mathrm{FAR}]$ is equivalent to the probability that $S_{n}$ stays at state $U_{F}$ (i.e. exceeding threshold $h$ ) under hypothesis $\mathcal{H}_{0}$.

According to [34], it states the transition probability matrix $\mathbf{P}$ always has a special eigenvector with only one eigenvalue $\lambda=1$ and the rest is zero. Thus, we can obtain the solution by re-elaborating the equation (22) into the matrix form as:

$$
\left[\begin{array}{cccc}
P_{00}-1 & P_{01} & \cdots & P_{0 F} \\
P_{10} & P_{11}-1 & \cdots & P_{0 F} \\
\vdots & \vdots & \ddots & \vdots \\
P_{F 0} & P_{F 1} & \cdots & P_{F F}-1 \\
1 & 1 & \cdots & 1
\end{array}\right]\left[\begin{array}{c}
\pi_{0} \\
\pi_{1} \\
\vdots \\
\pi_{F}
\end{array}\right]=\left[\begin{array}{c}
0 \\
0 \\
\vdots \\
0 \\
1
\end{array}\right] .
$$

By least squares estimation, the average false alarm rate can be determined by

$$
E_{\mathbf{P}}[\mathrm{FAR}]=\pi_{F}
$$

\section{The Expectation of missed detection ratio}

We define the missing detection probability as the probability that the detection delay is greater than or equal to a detection delay constraint $C$. The expectation $\left(E_{\hat{\mathbf{P}}}[\mathrm{MDR}]\right)$ of the missing detection probability is, starting from the initial state, the summation of probabilities that $S_{n}$ stays at a state other than state $U_{F}$ at time $C$. Let $p_{i}(s)$ denote the probability of the state variable at time $s$ and at state $i$. We set the initial condition for the transition probabilities as

$$
p_{i}(0)=\pi_{i}^{0},
$$

where $i \in\left\{0, U_{1}, \cdots, U_{F-1}\right\}$ and $p_{F}(0)=0$. By the iteration, at each $s$, the state probability vector is updated by the previous state probability vector in a matrix form as:

$$
\left[\begin{array}{c}
p_{0}(s) \\
p_{1}(s) \\
\vdots \\
p_{F-1}(s) \\
p_{F}(s)
\end{array}\right]^{T}=\left[\begin{array}{c}
p_{0}(s-1) \\
p_{1}(s-1) \\
\vdots \\
p_{F-1}(s-1) \\
p_{F}(s-1)
\end{array}\right]^{T} \hat{\mathbf{P}}
$$

and

$$
p_{F}(s)=0, \quad s \in\{0, C-1\} .
$$

Here the $p_{F}(s)$ at every $s$ of state $U_{F}$ is reset to zero for the next iteration since we only concern the missing detection case only. The expectation of missed detection ratio under the given delay constraint $C$ can be obtained as

$$
E_{\hat{\mathbf{P}}}[\mathrm{MDR}]=\sum_{i=0}^{F-1} p_{i}(C) .
$$

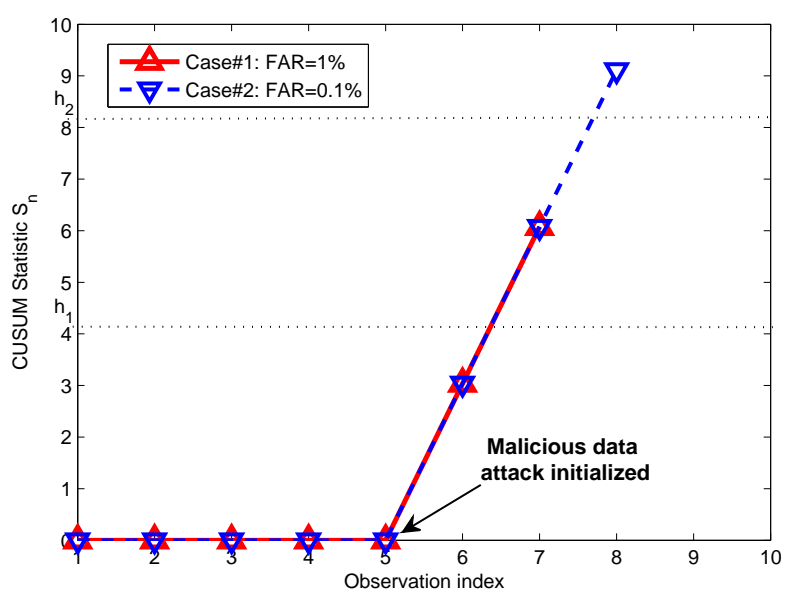

Fig. 2. The simulation of the adaptive CUSUM algorithm. The $x$-axis is the observation index $(n)$, and $y$-axis is the recursive CUSUM statistic $\left(S_{n}\right)$. Case 1 with FAR of $1 \%$ corresponds to $h_{1}$, and Case 2 with FAR of $0.1 \%$ corresponds to $h_{2}$. The proposed algorithm signals the alarm and then terminates the process at $T_{h}=7$ and 8 , respectively.

\section{Performance AnAlysis}

In this section, we present the analytical and numerical simulations to demonstrate the performance of the proposed scheme. This section is composed by two main sub-sections. The first sub-section demonstrates the performance of the proposed scheme from the simulated data. In other words, we heuristically configure the parameter and analyze the detection performance. The second sub-section involves both analytical and numerical results under the realistic power test systems by MATPOWER 4.0 package [21]. Without loss of generality, we assumes that the simulation has normalized sample rate ${ }^{2}$ and the static system ${ }^{3}$, Note that the adversary is able to inject the false power flow measurement at the random time.

\section{A. Simulation Results with Simulated Data}

Figure 2 illustrates the relation between the detection parameters $\left(S_{n}, h\right)$ and performance metrics $\left(\mathrm{FAR}, T_{D}\right)$. The number of measurements $m=4$. On the detector side, the detector has no information about the adversary statistical model, distribution, or any unknown. The adversary manipulates and injects the false data into the system at the random time. As shown in Figure 2, we consider that Case 1 with FAR of 0.01 and Case 2 with FAR of 0.0001 . The adversary becomes active and injects the false data at $n=6$. In other words, a change distribution is at $\tau=6$ from $\mathcal{N}\left(0, \boldsymbol{\Sigma}_{\mathbf{R}}\right)$ to $\mathcal{N}\left(\mathbf{a}_{n}, \boldsymbol{\Sigma}_{\mathbf{R}}\right)$,

\footnotetext{
${ }^{2}$ Since the measured noise is white Gaussian (independent over time), the performance of the quickest detection is depended on the number of observations. In other words, the decision time is related to the sampling rate, and the decision time is equivalent to the number of observation divided by the sampling rate.

${ }^{3}$ The reason we have a steady state or quasi-steady state system is that our algorithm can converge in very short time. For the PJM network, it is able to have state estimation for measurement of more than 2000 buses per minute [24]. From the simulation, we can see our algorithm converges around 100 samples. In other words, our algorithm can converge within a couple of seconds, during which the states can be considered at least quasi-steady.
} 

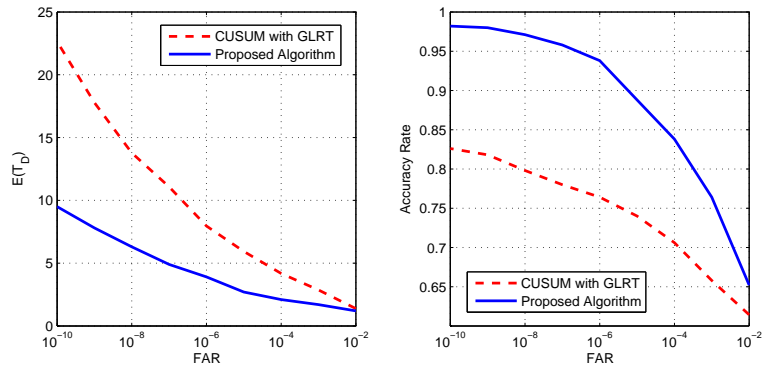

Fig. 3. The performance analysis of the adaptive CUSUM algorithm in comparison with CUSUM GLRT.

where $\mathbf{a}_{n}$ is unknown. For both cases, the curve of adaptive CUSUM statistic $\left(S_{n}\right)$ shows the sudden increase right after a change of distributions. The proposed algorithm quickly responses the abnormal event by signaling an alarm when $S_{n}$ passing the threshold. At the observation index 7, the threshold parameters $h_{1}$ and $h_{2}$ are corresponded to Case 1 and Case 2 , respectively. As a result, $h_{1}$ is less than $h_{2}$, because of the different FARs. For the smaller FAR, the stricter constraint that causes increasing the threshold; the higher requirement for system to declare the decision. ARL $\left(T_{h}\right)$ of the adaptive CUSUM algorithm is 7 and 8 at $S_{n}$ of 6.07 (Case 1) with $h=5.97$ and 9.11 (Case 2) with $h=8.19$, respectively. ARL $\left(T_{D}\right)$ of detection delay is 1 for cases 1 and 2 for the Case 2 in this simulation. The proposed algorithm is able to signal the alarm and terminates the process after the active false date attack.

Figure 3 shows the characteristics of the proposed algorithm by varying FAR for the accuracy rate and expected $\left(E\left[T_{D}\right]\right)$ of detection delay in comparison to that of the CUSUM GLRT. We run 5000 realizations for the simulation. FAR is vary from $10^{-10}$ to $10^{-2}$. The false data injection is begun at the 6th observation index. The accuracy rate in Figure 3(Right) represents the ratio of successful detection that the algorithm terminates the process and declares the existence of adversary after the 6th observation index (the actual attack index). As shown in the figure for both proposed scheme and the CUSUM GLRT, the stricter FAR is, the greater expected detection delay and higher detection accuracy we have. The expected detection delay of CUSUM GLRT seems to increase exponentially while that of proposed scheme steady raises as FAR decreases. $E\left[T_{D}\right]$ of the proposed scheme has the average $50 \%$ less than that of CUSUM GLRT. We also obtain the better accuracy rate as FAR decreases. By giving the sufficiently low FAR, the proposed scheme is able to reach the accuracy above $95 \%$ while CUSUM GLRT struggles it below 83\%. Therefore, the proposed scheme outperforms the CUSUM GLRT in terms of shorter decision time and higher detection accuracy. The simulation result also shows the tradeoff between the detection delay, false alarm and accuracy rate. The smaller FAR causes higher delay but better accuracy, i.e., the system needs to spend more observations for making a decision.
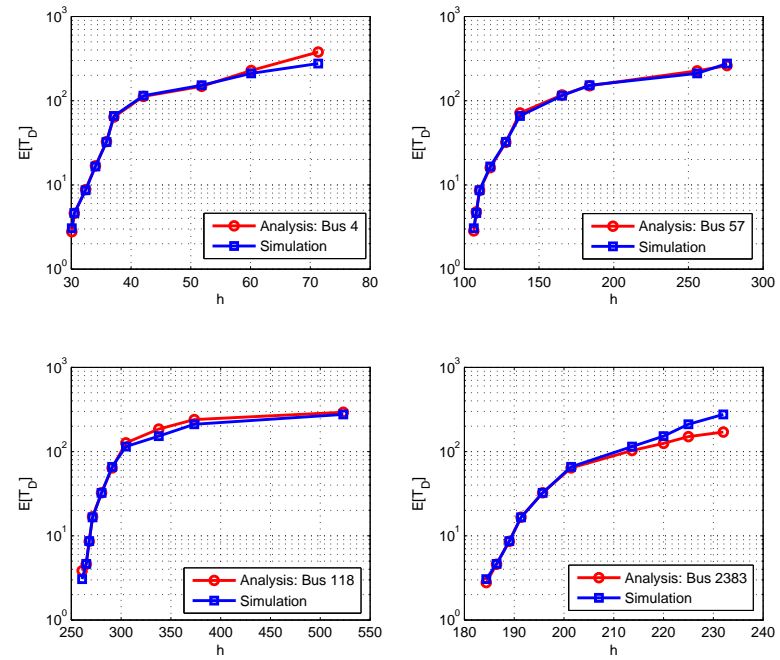

Fig. 4. The expectation $E\left[T_{D}\right]$ of detection delay for different IEEE Bus test systems.

\section{B. Simulation Results with MATPOWER 4.0}

For the experiment setup of this subsection, we first apply the analytical model to theoretically analyze the performance of the detection system for guiding the system parameter configuration. Then, we use the parameter from the theoretical analysis to confirm the accuracy of the analysis in the first half of the subsection, and then demonstrate the performance of the detection system in the second half of the subsection.

1) Accuracy of the analytical model: In this section, the power flow data for all simulations are generated by MATPOWER 4.0 instead of random independent variables in the previous subsection. MATPOWER 4.0 is a Matlab simulation tool for solving power flow and optimal power flow problems. It provides realistic power flow data and test systems that uses widely in research-oriented study as well as in practice. We consider 4 popular IEEE test systems from the MATPOWER 4.0 package. Case 1 is the IEEE 4-bus test system, which has 2 generators for 4 measurements; Case 2 is the IEEE 57bus test system, which has 7 generators for 80 measurements; Case 3 is the IEEE 118-bus test system, which has 54 generators for 186 measurements; and Case 4 is the IEEE 2383-bus test system, which has 326 generators for 2896 measurements. The analytical performance measures and the simulation results are compared under same setting and input data to examine. Hence, by using power flow data sets with 4 different study cases from MATPOWER 4.0, the performance indices ( $\left.E[\mathrm{FAR}], E[\mathrm{MDR}], E\left[T_{D}\right]\right)$ comparisons between the analytical and simulation result can be conducted. With the parameter from the theoretical analysis, the performance indices are simulated so that we can properly configure the proposed algorithm for the guaranteed performance. Notice that both theoretical analysis and simulation are plotted together to confirm accuracy of analysis and demonstrate the performance.

Figure 4 gives us an insight of the relationship between 

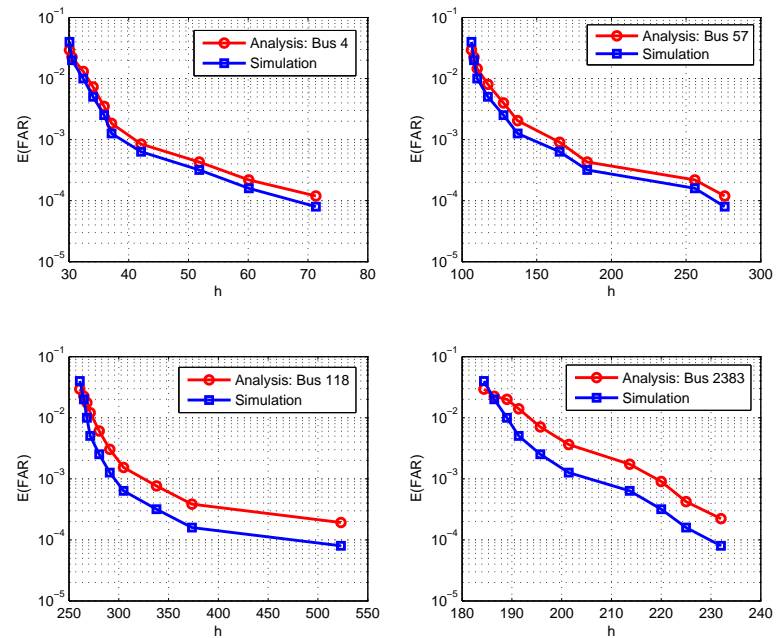

Fig. 5. The expectation $E[\mathrm{FAR}]$ of false alarm rate for different IEEE Bus test systems.

the system parameters $h$ and the detection delay $E\left[T_{D}\right]$ of the proposed scheme. The higher the threshold, the larger the delay. Also shown in Figure 4, both analytical and simulation results are matched closely in all IEEE 4-bus, 57-bus, 118-bus test systems. The maximum difference between the analysis and simulation is around $2 \%$ in the case of IEEE 2383-bus test system.

The numerically examination is presented for understanding the impact of the fundamental performance metric FAR on system parameters $h$ of the proposed scheme. As shown in Figure 5 , the analytical and simulation result are close. Notes that the logarithmic scale is used in the figure for the vertical axis. In cases of IEEE 4-bus and 57-bus test systems, the difference percentage between the analysis and simulation is very small and near zero. However, as the number of bus increases (total number of active power flow measurement increases, too), the maximum difference percentage is about $8 \%$ in IEEE 2383-bus test system. More measurements can cause the larger variance when we try to calculate the covariance for computing $\mathbf{R}$. From the figure, we also can observe that a larger $h$ yields a smaller false alarm rate as expected.

The analytical result of $E[\mathrm{MDR}]$ is demonstrated under 2 scenarios of the delay constraints, in which $C=7$ and $C=18$. The result is shown in Figure 6 that helps us study the impact of the missed detection ratio on $h$ of the proposed scheme. The logarithmic scale is used in the figure for the vertical axis. From the figure, the larger constraint $C$ results smaller expectation of missed detection ratio as expected. In other words, the probability of detection rises if we allow to increase the cost of longer delay. We also compute the mean of expected missed detection ratio as the base line, in comparison with the analytical results for 4 different IEEE test systems. The trend of analysis follows the base line closely. However, as the number of active power flow measurement increases, the gap between them becomes obvious, especially, in case of IEEE 2383-bus test system,
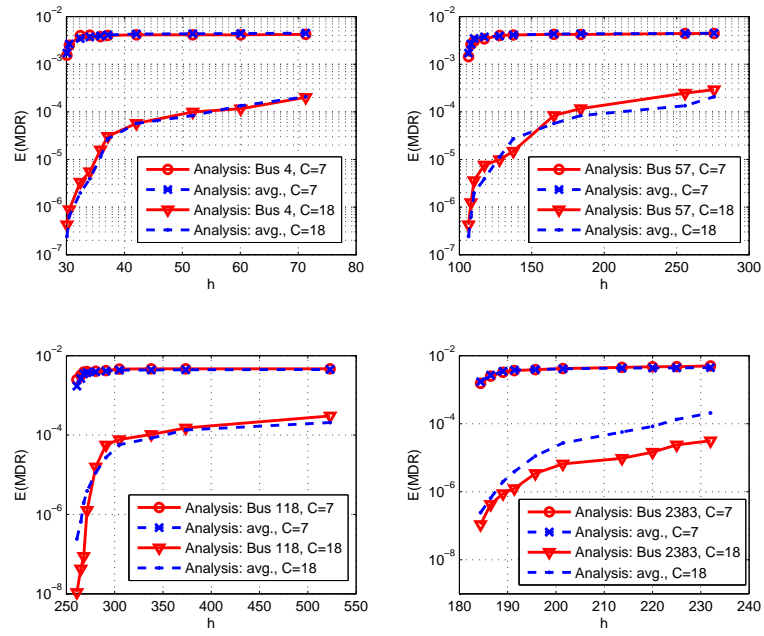

Fig. 6. The expectation $E[\mathrm{MDR}]$ of missed detection ratio for different IEEE Bus test systems.

the maximum difference percentage is obtained around $10 \%$. More measurements can cause the larger variance when we try to calculate the covariance for computing $\mathbf{R}$. In addition, the smaller $h$ is, the better the expectation of missed detection ratio that corresponds to the result of expected false alarm rate in Figure 5 as the tradeoff.

2) Detection with performance guarantee: From Figure 4-6, we demonstrate the performance metrics with different $h$. It also helps us to configure the system parameter $h$ for guaranteed performance under three fundamental metrics. For each different IEEE test system, we can select the proper configuration of $h$ from the reasonable range to satisfy the desired performance constraints. For examples, the configuration of $h$ is set to 135 for IEEE 57-bus test system; the analytical model of the proposed scheme shows that the expectation of the false alarm rate of 0.001 , the expectation of detection delay of 20 , and the expectation of missed detection ratio of 0.00005 under the delay constraint $C=18$. In addition, if we wish to have a certain level of detection probability, we can compute the numerical value of detection probability from Figure 4; with its corresponding $h$, we can explicitly determine the cost of detection delay from Figure 4 and the tradeoff for the false alarm rate from Figure 5. The above analysis can be extended to other IEEE power systems in a similar way.

In Figure 7, we show the CUSUM statistics $S_{n}$ over observation index $n$ for the IEEE 4-bus, 57-bus, and 118bus test systems. For the simulation setup, we considers that the false alarm rate of 0.01 is presented, and the active false data injection attack is initialized after the observation index 15. For the simulation results, in the IEEE 4-bus test system, the system is alarmed the after 24 observations with the corresponding detection threshold of 34.51 ; the detection delay is 9 . In the IEEE 57-bus test system, the system is alarmed the after 37 observations with the corresponding detection threshold of 133.52 and the detection delay of 22 . In the IEEE 118-bus test system, the system is alarmed the after 

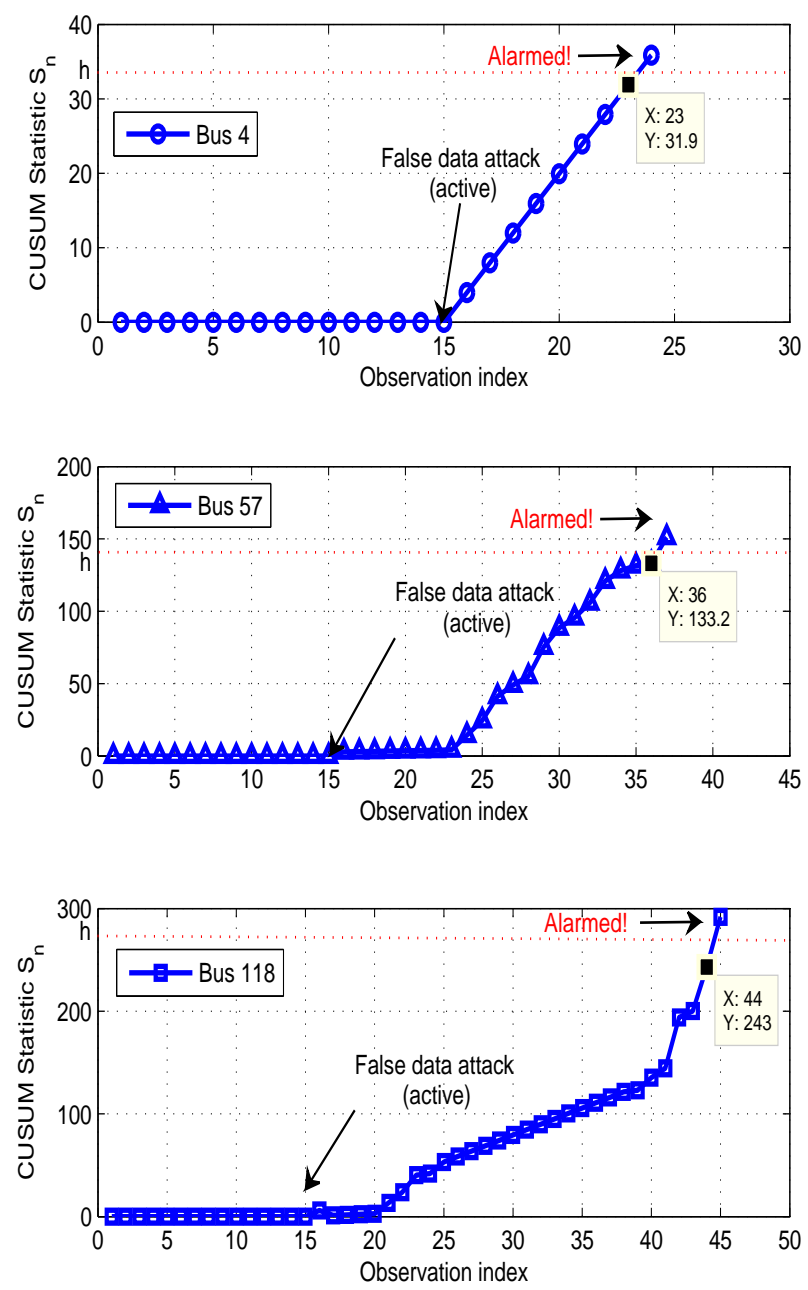

Fig. 7. The detection simulation of the adaptive CUSUM algorithm with MATPOWER 4.0 power-flow measurements for the IEEE 4-bus test system, IEEE 57-bus test system, and IEEE 118-bus test system. The $x$-axis is the observation $(n)$, and $y$-axis is the recursive CUSUM statistic $\left(S_{n}\right)$. The proposed algorithm signals the alarm and then terminates the process at $T_{h}=24,37$, and 45 , respectively.

45 observations with the corresponding detection threshold of 283.14; the detection delay in this test system is 30 . As expected, the simulation also shows that the detector need more observations to make the decision, when the number of the power-flow measurements and buses increases. Notices that the numerical results of each IEEE test system in Figure 7 is corresponded to our analytical results, which are presented in Figure 4-6.

\section{CONCLUSION}

In this paper, we propose the adaptive CUSUM algorithm for defending false data injection attack in smart grid networks. We successfully derive a detection model with considering the existence of the unknown, and then develop an analytical model that can guide us configure the detection system for performance guarantee based on the fundamental detection requirements. Our proposed scheme for smart grid state estimation composes two interleaved steps: i) introduces the unknown variable solver technique based on Rao Test, and ii) applies the multi-thread CUSUM algorithm for determining the possible existence of adversary as quickly as possible without violating the given constraints. Furthermore, we develop the Markov chain based analytical model to characterize the behavior of our proposed scheme. We can quantitatively study the system parameters to achieve the guaranteed detection performance in term of three fundamental metrics $(E[\mathrm{FAR}]$, $E[\mathrm{MDR}]$, and $\left.E\left[T_{D}\right]\right)$. The analytical and numerical simulation results have shown that the proposed scheme is efficient in terms of detection accuracy and minimum detection delay. Overall, the proposed scheme is able to achieve the important objectives of smart grid security in terms of real-time operation and security requirement.

In future work, we further investigate the optimality of a joint attack detection and state estimation in smart grid. When an attacker occurs in the power network, the ultimate objective of the network operator is beyond a reliable detection of the attack. In fact, detecting the attack will be used as an intermediate step towards obtaining a reliable estimate about the injected false data, which in turn facilitates eliminating the disruptive effects of the false data. Assuring good estimation performance is the core of estimation and detection problem in the smart grid networks. To account for the significance of estimation quality, we can define an estimation performance of measure and seek to the optimize it while ensuring satisfactory of the detection performance. The objective is to minimize the estimation-related cost subject to appropriate constraints on the tolerable levels of detection errors. This approach can provide the operator with the freedom to strike desired balance between estimation and detection qualities. Other future work can include the analysis of load/generation disruption and joint consideration with PMU.

\section{REFERENCES}

[1] U.S. Department of Energy (DOE), "The smart grid: an introduction," U.S. Department of Energy Book: Smart Grid Series, September 2012.

[2] E. Hossain, Z. Han, and V. Poor, Smart grid communications and networking, Cambridge University Press, UK, 2012.

[3] Dae-Hyun Choi and Le Xie,"Ramp-induced data attacks on look-ahead dispatch in real-time power markets," IEEE Transactions on Smart Grid, vol. 4, no. 3, pp. 1235-1243, 2013.

[4] D. Kundur, X. Feng, S. Liu, T. Zourntos, and K. L. Butler-Purry, "Towards a framework for cyber attack impact analysis of the electric smart grid," in Proceedings of IEEE Conference on Smart Grid Communications, October 2010

[5] S. McLaughlin, B. Holbert, A. Fawaz, R Berthier, and S. Zonouz, "A multi-sensor energy theft detection framework for advanced metering infrastructures," IEEE Journal on Selected Areas in Communications, vol. 31, no. 7, pp. 1319-1330, 2013.

[6] M. Ozay, I. Esnaola, F.T.Y. Vural, S.R. Kulkarni, and H.V. Poor, "Sparse attack construction and state estimation in the smart grid: centralized and distributed models," IEEE Journal on Selected Areas in Communications, vol. 31, no. 7, pp. 1306-1318, 2013.

[7] Chun-Hao Lo and N. Ansari, " CONSUMER: a novel hybrid intrusion detection system for distribution networks in smart grid," IEEE Transactions on Emerging Topics in Computing, vol. 1, no. 1, pp. 33-44, 2013. 
[8] I. Matei, J. S. Baras, and V. Srinivasan, "Trust-based multi-agent filtering for increased Smart Grid security," in Proceedings of 20th Mediterranean Conference on Control and Automation, July 2012.

[9] Y. Huang, M. Esmalifalak, Yu Cheng, Husheng Li, Kristy A. Campbell and Zhu Han, "Adaptive quickest estimation algorithm for smart grid network topology error," IEEE Systems on Journal, vol. PP, no. 99, pp. 1-11, July 2013.

[10] Y. Huang, M. Esmalifalak, H. Nguyen, R. Zheng, Z. Han, Husheng Li, Lingyang Song, "Bad data injection in smart grid: attack and defense mechanisms," IEEE Communications Magazine, vol. 51, no. 3, pp. 2733, January 2013

[11] M. Talebi, C. Li , and Z. Qu, " Enhanced protection against false data injection by dynamically changing information structure of microgrids," IEEE 7th Sensor Array and Multichannel Signal Processing Workshop, June 2012.

[12] J. Lin, W. Yu, X. Yang , G. Xu, and W. Zhao, "On False Data Injection Attacks against Distributed Energy Routing in Smart Grid," in Proceedings of IEEE/ACM 3rd International Conference on CyberPhysical Systems, April 2012.

[13] H. V. Poor and Q. Hadjiliadis, Quickest Detection, Cambridge University Press, 2008.

[14] Y. Huang, Lifeng Lai, Husheng Li, W. Chen, and Z. Han, "Online quickest multiarmed bandit algorithm for distributive renewable energy resources," in Proceedings of IEEE Conference on Smart Grid Communication, Taiwan, November 2012.

[15] M. Basseville and I. Nikiforov, Detection of Abrupt Changes: Theory and Applications, Englewood Cliffs: Prentice-Hall, NJ, April 1993.

[16] H. Li, C. Li, and H. Dai, "Collaborative quickest detection in ad hoc networks with delay constraint - Part I: Two-node network," IEEE Information Sciences and Systems 2008, pp. 594 - 599, Princeton, NJ, March 2008.

[17] J. Tang, Y. Cheng, and W. Zhuang, "An analytical approach to realtime misbehavior detection in IEEE 802.11 based wireless networks", in Proceedings of IEEE International Conference on Computer Communications, April 2011.

[18] Y. Huang, H. Li, K. A. Campbell, and Z. Han, "Defending false data injection attack on smart grid network using adapative CUSUM test," in Proceedings of IEEE Conference on Information Sciences and Systems, March 2011.

[19] A. Abur and A. G. Exposito, Power System State Estimation: Theory and Implementation, Marcel Dekker, Inc., 2004.

[20] A. J. Wood and B. F. Wollenberg, Power Generation, Operation, and Control, Wiley New York et al., 1996.

[21] R. D. Zimmerman, C. E. Murillo-Schnchez, and R. J. Thomas, "MATPOWER steady-state operations, planning and analysis tools for power systems research and education," IEEE Transactions on Power Systems, Vol. 26, No. 1, pp. 12-19, February 2011.

[22] F. C. Schweppe, J. Wildes, and D. B. Rom, "Power system static state estimation ," IEEE Transactions on Power Apparatus and systems, vol. 89, no. 1, pp. 120-135, January 1970.

[23] J. Casazza and F. Delea, Understanding Electric Power Systems, IEEE Press Understanding Science and Technology Series, A John Wiley and Sons, Inc., 2010.

[24] A. L. Ott, "Experience with PJM market operation, system design, and implementation," IEEE Transactions on Power Systems, Vol. 18, No. 2, pp. 528-534, May 2003.

[25] E. S. Page, "Continuous inspection schemes," Biometrika, Vol. 41, No. 1/2, pp. 100-115, July 1954 .

[26] M. He and J. Zhang, "Fault detection and localization in smart grid: A probabilistic dependence graph approach," in Proceedings of IEEE Conference on Smart Grid Communications, October 2010.

[27] G. Lorden, "Procedures for reacting to a change in distribution," Annals of Mathematical Statistics, Vol. 42, No. 6, pp. 1897-1908, July 1971.

[28] A. De Maio and S. Iommelli, "Coincidence of the Rao Test, Wald Test, and GLRT in partially homogeneous environment", IEEE Letter on Signal processing, Vol. 15, No. 1, pp. 385-388, April 2008.

[29] K. J. Sohn, "Parametric tests for multichannel adaptive signal detection," Ph.D Dissertation from Stevens Institute of Technology, December. 2007.

[30] A. D. Maio, "Rao test for adaptive detection in Gaussian interference with unknown covariance matrix," IEEE transactions on signal processing, Vol. 55, No. 7, pp. 3577-3584, July 2007.

[31] S. M. Kay, Fundamentals of Statistical Signal Processing: Detection Theory, Englewood Cliffs, NJ: Prentice-Hall, 1998.

[32] M. R. Chmielewski and J. W. Grzymala-BusseJ, "Global discretization of continuous attributes as preprocessing for machine learning," International Journal of Approximate Reasoning, Vol. 15, No. 4, pp. 319-331, November 1996.
[33] Y. Q. Chen and K. L. Moore, "Discretization schemes for fractionalorder differentiators and integrators", IEEE Transactions on Circuits and Systems I: Fundamental Theory and Applications, Vol. 49, No. 3, pp. 363-367, March 2002.

[34] D. Gamerman and H. F. Lopes, Markov Chain Monte Carlo: Stochastic Simulation for Bayesian Inference, Boca Raton, FL: Chapman and Hall/CRC, 2006. 